\title{
Recherche et innovation citoyenne par la Recherche Action Participative
}

\section{Citizen research and innovation by Participatory Action Research}

\author{
Sylvie Blangy ${ }^{1}$, Bertrand Bocquet ${ }^{2}$, Cyril Fiorini ${ }^{3}$, Jean-Marc Fontan ${ }^{4}$, Martine Legris ${ }^{5}$, \\ Christian Reynaud ${ }^{6}$
}

\footnotetext{
${ }^{1}$ Centre d'Ecologie Fonctionnelle et Evolutive, UMR 5175, CNRS, Montpellier, France, Sylvie.blangy@cefe.cnrs.fr

${ }^{2}$ Laboratoire d'Histoire des Technosciences en Société, EA 3716. Cnam, Université de Lille, Paris, France, Bertrand.Bocquet@univ-lille1.fr

${ }^{3}$ Laboratoire d'Histoire des Technosciences en Société, EA 3716, Cnam, Paris, France, cyril.fiorini@cnam.fr

${ }^{4}$ Centre de recherche sur les innovations sociales, Université du Québec à Montréal (UQAM), Canada, fontanjeanmarc@gmail.com

${ }^{5}$ Centre d'Etudes et de Recherches Administratives, Politiques et Sociales, UMR8026, CNRS, Université de Lille, Lille, France, mart.revel@gmail.com

${ }^{6}$ Laboratoire Interdisciplinaire de Recherche en Didactique, Éducation et Formation, EA 3749, Université de Montpellier et Université Paul Valéry de Montpellier, Montpellier, France, christian.reynaud@umontpellier.fr
}

* Ordre alphabétique, tous les auteurs ont également contribué à la recherche et à la rédaction de l'article

RÉSUMÉ. La recherche scientifique est sollicitée pour investiguer les grands défis sociétaux posés par nos sociétés contemporaines. Une approche transversale est requise se traduisant par le développement de recherches à différentes interfaces et notamment celles liant les sciences expérimentales, sociales et humaines. Cependant, ces avancées ne prennent pas suffisamment en compte les problématiques citoyennes directement en prise avec ces défis. Nous montrons que la Recherche Action Participative permet de développer des méthodologies de recherche propres pourvu qu'elle puisse se déployer dans des espaces hybrides permettant la rencontre et le travail pérennes des différentes parties prenantes d'une recherche : chercheurs, associatifs, praticiens, etc. Nous relatons l'expérience menée par le GDR CNRS PARCS dont le fonctionnement s'apparente à un laboratoire de recherche hors-mur, l'impact qu'il a eu sur ses membres notamment au travers de cycles de formation et les conclusions positives qui en sont issues.

ABSTRACT. Scientific research is requested for investigating hard societal challenges brought by our contemporary societies. A transversal approach is required by the development of interdisciplinary research, especially at the interface between experimental, social and human sciences. However this approach remains insufficient and minimizes citizens' concern about these challenges. We show in this paper that Participatory Action Research allows us to develop original research methods if it can work in hybrid stable forums. These spaces allow us to meet and to work with stakeholders of a research project (researchers, non-profit organization members, practitioners, etc.) in the medium or long term. Such a forum was created in 2013 as a formal research group of the CNRS named Participatory Action Research and Citizen Sciences (GDR CNRS PARCS). This forum is like a research laboratory without walls. We describe our experiences led in this laboratory, the impact on the members due to the development of training and the positive conclusions that we can obtain for the development of such approaches.

MOTS-CLÉS. Recherche Action Participative, Innovation sociale, Participation, Société de la connaissance, Capacitation, Co-production des savoirs, Sciences en société, Démocratie technique.

KEYWORDS. Participatory Action Research, Social innovation, Public engagement, Knowledge society, Empowerment, Co-production of knowledge, Sciences in society, Technical democracy.

\section{Introduction}

Nous assistons depuis plusieurs années à une reconfiguration importante de l'espace de la recherche scientifique notamment européen, avec des traductions sur les plans nationaux. Cette reconfiguration tend, d'une part, à prendre davantage en compte la complexité du monde dans lequel nous évoluons, et, d'autre part, d'investiguer et de proposer des solutions au regard des grands défis sociétaux que posent nos sociétés contemporaines. Ces défis présentent une dimension incommensurable plus ou moins marquée qui nécessite l'acquisition de nouvelles connaissances et l'élaboration de solutions originales 
en liant les sciences expérimentales aux sciences humaines et sociales, mais également des préoccupations institutionnelles, économiques et citoyennes. Nous nous intéresserons plus spécifiquement à cette dernière dimension qui est encore peu prise en compte actuellement dans les politiques de recherche. Dans un premier temps, nous aborderons assez largement la problématique dans laquelle nous nous situons. Le contexte général est celui de la volonté de développer de nouvelles formes de démocratie et notamment la démocratie délibérative [GIR 13]. Il influence nécessairement le champ de la recherche scientifique et de l'innovation au travers de ce que l'on appelle la démocratie technique [LEQ 14], appelée elle-même à se renouveler sous le terme par exemple des «Sciences en société» [BON 13], [VAN 17]. Nous décrirons et catégoriserons le foisonnement d'expériences de l'interface sciences-société pour dégager la spécificité de la Recherche Action Participative (RAP) développée au sein du Groupement de recherche du CNRS «Participatory Action Research and Citizen Sciences » (GDR PARCS). Ce groupement constitue lui-même une expérience intéressante dont l'originalité par rapport au sens classique des GDR, est de constituer un laboratoire expérimental « hors-mur » sur le mode des Living Lab [DEL 14]. L'objectif principal de ce laboratoire est d'élaborer des méthodologies originales permettant la co-construction et la réalisation de recherches avec pour finalité, la co-production de connaissances, de savoirs, de normes et d'innovations.

\section{Les démarches participatives dans la production des connaissances scientifiques}

Les relations sciences-société ne sont pas nouvelles et concernent aussi bien des intérêts particuliers (entreprises par exemple) que l'intérêt général (amateurs de science par exemple). Par contre, la nature de ces relations change d'échelle depuis les années 1980 avec un nouveau régime de production technoscientifique basé sur une forte standardisation des savoirs [RUA 17]. L'importance de la recherche scientifique conduit à optimiser l'interface entre acquisition de nouvelles connaissances et élaboration de solutions ou d'offres de nouveaux produits par l'innovation. Les objectifs sont ceux de la croissance et de la compétitivité.

En parallèle, le contexte politique contemporain est marqué par l'essor d'une demande parfois diffuse de participation aux choix scientifiques et techniques, reconnue dans le droit européen et national et qui s'est concrétisée notamment dans des dispositifs institutionnels (débat public, conférence de citoyens, etc.). Depuis une quinzaine d'années se sont multipliées en France comme à l'international les études consacrées à la démocratie participative ou délibérative et à des dispositifs relevant de ce nouvel impératif délibératif [BLO 02]. Cette demande sociale marquerait un «tournant délibératif », basé sur un système dialogique [HAB 87], [COH 89], [MAN 85]. Plusieurs modèles normatifs proposent dans sa continuité, de valoriser l'implication des citoyens dans les procédures de décision et de théoriser les moyens de faire converger la « démocratie de la confiance » de Rosanvallon [ROS 06], le modèle de la « démocratie dialogique » [CAL 01], la « démocratie du public » [MAN 95] et la «démocratie écologique» [BOU 11]. Malgré des formes différentes, ces approches partagent la nécessité de proposer des espaces d'interactions s'appuyant sur des procédures hybrides où les acteurs (politiques, scientifiques et "profanes») viennent confronter leurs mondes communs et débattre des thématiques scientifiques pour mener l'enquête sociale [DEW 27].

La majeure partie des dispositifs participatifs actuellement pratiqués en France dans le cadre de l'action publique vise la construction d'un consensus entre toutes les parties prenantes. Ils ont émergé suite aux mobilisations contre la construction de la ligne TGV Sud Est, pour sortir de situations bloquées, où les protagonistes campaient sur des positions radicalement opposées concernant l'implantation d'infrastructures [FOU 01]. L'objectif consiste ainsi à produire une réflexion collective dans certains cas, un avis dans d'autres, qui soient partagés par les participants.

Dans un contexte où les controverses scientifiques préoccupant le citoyen se multiplient (nucléaire, OGM, changements climatiques, nanotechnologies, transhumanisme...) et face à la complexification croissante des modes de gouvernance de la science et de l'innovation, les pouvoirs publics ont progressivement mis à l'agenda politique le développement de dispositifs de délibération qui 
permettent de débattre des options scientifiques et techniques en prenant en compte différents systèmes de valeurs et différentes visions du monde [BOU 05]. Dès lors, la réflexion sur les conditions et degrés d'inclusion du public à la définition de l'action publique, mais aussi à la détermination des cadrages et des modalités des procédures de participation, semble indispensable. Le véritable enjeu démocratique réside dans les moyens donnés aux individus de produire des savoirs et de les légitimer dans l'espace public, plutôt que dans la seule capacité à s'exprimer. Il ne s'agit donc pas d'étudier les formes et gradients de la démocratie participative, mais de s'intéresser également aux modalités de co-production de la recherche. Non dénuée d'ambiguités, cette tendance s'incarne dans différentes modalités de collaboration. On parlera suivant les époques et les auteurs de recherche-action, de sciences citoyennes, d'enquête collaborative ou de recherche participative. Il n'existe pas en la matière de cadre théorique stabilisé qui définisse clairement les différents modèles et méthodes de la recherche participative, bien que la littérature sur la question soit foisonnante [CHA 89], [ANA 07].

La production scientifique en France reste encore largement le fruit d'un travail professionnalisé exercé au sein d'un champ académique dont l'autonomie est recherchée par une large partie des communautés scientifiques [COM 15] tout en restant néanmoins dépendante des pouvoirs politiques et économiques [PES 03]. Les critères dominants de scientificité attendus des résultats de recherche par les communautés disciplinaires induisent ainsi une certaine étanchéité du processus de recherche scientifique auquel ne peuvent accéder que les individus reconnus comme légitimes pour y intervenir (obtention d'un diplôme de doctorat, obtention de la «qualification » comme droit de passage pour postuler aux emplois d'enseignants-chercheurs, recrutement par les pairs...).

Si la recherche publique française s'inscrit majoritairement dans un tel modèle de production et de régulation scientifique, il ne doit pas néanmoins masquer le développement d'une diversité de processus mis en œuvre dans la production des connaissances et des savoirs qui visent également un objectif de scientificité tout en déployant des modalités de travail différentes. En effet, « la production des savoirs scientifiques, par exemple, ne relève pas seulement de professionnels » [PES 06]. Nonobstant une grande diversité de pratiques, d'objectifs, de justifications et de résultats, ces dynamiques de recherche alternatives se caractérisent par la possession d'un élément commun : celui d'ouvrir le processus de production scientifique à des acteurs non-scientifiques [CAL 01], [PIM 11][REV 14], [AUD 15], [HOU 16].

De nombreuses tentatives de classements des différents niveaux de participation ont été effectuées selon différents critères. Les travaux menés ont davantage porté sur l'observation des processus à l'œuvre en élaborant une typologie des différentes pratiques de collaboration ou des différentes modalités de « co-construction des savoirs » [ANA 07].

Une étude s'est penchée sur une classification en fonction des disciplines scientifiques à partir d'une étude scientométrique basée sur des articles du Web of Science (WoS). Des mots clés ont été identifiés qui font apparaître que la dénomination de Citizen science (Science citoyenne) émerge dans les années 1990 avec un tournant quantitatif dans les années 2010. D'autres terminologies sont également employées comme Observation Volontaire, Surveillance par les Communautés, Science Participative, Épidémiologie Populaire, etc. Cette augmentation est mise en rapport avec le développement, à cette époque, des plateformes numériques Web qui permettent d'agréger de très nombreux contributeurs aux recherches scientifiques [KUL 16]. Les articles dont il s'agit ici sont principalement dédiés à la collecte de données par des non-scientifiques. Néanmoins, il existe d'autres orientations moins fréquentes basées sur des pratiques plus locales ou plus militantes dont la trace dans les articles est plus difficile à identifier. Les termes employés sont alors Système d'Information Géographique, Technoscience Civique, Audit Citoyen, Contrôle Environnemental Citoyen, Science Urbaine, Science du DIY ${ }^{1}$, etc. Les domaines de recherche principaux représentés sont (i) les sciences naturelles avec principalement la conservation, la biodiversité et le changement climatique, (ii) la géographie avec la notion

\footnotetext{
${ }^{1}$ Do It Yourself
} 
d'information géographique volontaire, (iii) les sciences sociales et politiques centrées sur le phénomène de la participation et de l'engagement public, et (iv) la santé. Un autre critère pourrait être centré sur le degré de participation et d'implication des citoyens dans les projets de recherches participatives qui peuvent affecter significativement le déroulement d'un projet ainsi que les résultats attendus par chacune des parties prenantes. Un article de synthèse défini le terme de Participation Publique en Recherche Scientifique (PPRS) qui transcende les différents champs disciplinaires et des pratiques plus traditionnelles des sociétés savantes [SHI 12] ${ }^{2}$. Les projets de recherche analysés sont classés en cinq modèles caractéristiques regroupés en fonction du degré croissant de participation du public: contractuel, contributif, collaboratif, co-créatif et collégial. Une telle description est intéressante dans la mesure où, selon les auteurs, le cadre d'analyse ainsi défini peut être appliqué à des projets issus de toutes disciplines.

Nous avons choisi de caractériser les recherches menées sur l'interface sciences et participation en identifiant le promoteur de la recherche indépendamment des disciplines abordées ou des cadres définis pour le processus de recherche [SAV 17]. En d'autres termes, le critère discriminant à partir duquel différencier les pratiques et tenter de les classifier est soumis à la question de savoir qui formule la problématique de recherche. Dans une telle démarche, trois familles principales apparaissent : (i) une première où la problématique de recherche est issue des chercheurs, (ii) une deuxième où ce sont des non-chercheurs qui sont porteurs de la question de recherche et (iii) une troisième intermédiaire où une demande de recherche n'est pas clairement définie et fait l'objet d'une négociation entre les acteurs d'un projet.

Le premier cas fait référence principalement à la citizen science que l'on traduit en France plutôt par le terme de "sciences participatives ». Ce mode de recherche fait appel à des publics bénévoles intéressés à contribuer à un projet par l'apport de données essentiellement d'observation. Il connaît actuellement un développement constant qui bénéficie des progrès dans les technologies de l'information et de la communication, essentiellement autour de l'Internet et des applications mobiles. Ces nouveaux outils augmentent non seulement la visibilité des projets permettant le recrutement de nombreux bénévoles, mais aussi leur fonctionnalité et leur accessibilité. L'impact scientifique de ces projets est de plus en plus robuste avec le développement de protocoles appropriés, de formations en présentiel ou en virtuel pour les bénévoles et la conception d'outils statistiques et numériques performants permettant d'identifier des biais ou des erreurs de mesure dans les données, d'identifier des paramètres et de les cartographier. L'impact social est lui aussi significatif par l'exploitation des résultats de ces recherches, aussi bien au niveau des groupes sociaux impliqués dans les projets que d'organismes gouvernementaux ou privés [BON 14]. Ces impacts sont de différents ordres en fonction du domaine de recherche concerné. Par exemple, ce peut être la mise en place de zones protégées, des activités agricoles ou de pêche s'orientant vers plus de soutenabilité, une cartographie dynamique de la qualité de l'air, etc.

À l'opposé du cas précédent, une deuxième famille peut être identifiée lorsque la question de recherche est portée par une organisation de la société civile à but non-lucratif. L'intérêt de ces organisations pour la recherche scientifique est plus récent, à la différence du monde économique [AUD 11]. La demande de recherche est généralement formulée par des groupes de citoyens constitués en collectifs ou en associations et correspond à un besoin pour l'organisation, de développer des connaissances ou des expertises sur des problématiques qui lui sont propres [EPS 01]. Les résultats obtenus peuvent servir à éclairer des plaidoyers à destination du public ou de décideurs publics pour des organisations dont la mission principale est d'ordre militante. Nous pouvons alors parler de «sciences impliquées» [COU 15]. Ils permettent de développer des connaissances sur des sujets qui ne sont pas traités par la recherche scientifique, par exemple, sur des questions transversales telles que les impacts de projets technoscientifiques ou le développement d'outils alternatifs d'intérêt général, par exemple, des logiciels informatiques. Dans ce cas, l'engagement des acteurs de la société civile est

\footnotetext{
${ }^{2}$ Voir l'article « Analyse Citoyenne des Données » dans le même numéro spécial
} 
important mais la difficulté provient essentiellement des moyens à trouver pour financer les recherches. Des évolutions se font jour pour améliorer ces capacités d'intervention avec le développement de financements participatifs (crowdfunding) ou par le dépôt de projets de recherche vers des bailleurs publics comme des collectivités régionales, l'Agence Nationale de la Recherche (ANR) en France, le programme cadre de recherche et développement Horizon 2020 en Europe ou de fondations privées comme la Fondation de France qui ouvrent leurs programmes à destination d'organisation de la société civile à but non-lucratif.

Le troisième cas est beaucoup plus récent et montre une évolution importante qu'entretient la société dans son ensemble avec le monde de la recherche scientifique. Il s'agit de co-construire une problématique entre une équipe de chercheurs et les membres d'une organisation. En France, les termes de « recherche participative» ou "recherche action participative» sont généralement utilisés pour décrire cette forme de recherche. Les développements principaux se font au travers de projets pluriannuels financés par des programmes spécifiques ou au travers de dispositifs tels que les Boutiques des Sciences [SAV 17]. Il est important de noter que l'élaboration de la question scientifique à traiter est l'élément clé du projet. Elle passe par une phase de traduction-négociation entre les parties prenantes dont la durée n'est pas négligeable puisqu'elle dépasse parfois les six mois. La question conditionne également la méthodologie qui sera déployée pour la recherche, par exemple, en garantissant un déroulement de projet dans l'équité et la bienveillance entre les partenaires. C'est sans doute la raison pour laquelle un accompagnement du processus par un tiers peut être souhaité. Il est intéressant de constater que cette forme originale de faire de la recherche sur des questions d'intérêt général, orientation généralement réservée à la recherche scientifique publique, acte en quelque sorte l'importance que l'ensemble de la société accorde à la méthode scientifique pour aborder une meilleure compréhension du réel.

Les démarches qui nous intéressent ici et que l'on peut considérer comme des processus de «coproduction des savoirs » se fondent sur l'implication du « groupe concerné » par l'objet de la recherche dans l'élaboration de la problématique de recherche, la production des savoirs, mais aussi leur évaluation [CAL 98]. En suivant la définition proposée par Michel Callon de la co-production des savoirs comme le modèle le plus engagé de démocratie technique [CAL 98] $]^{3}$, les individus impliqués dans le processus de recherche scientifique reconnaissent mutuellement la légitimité des savoirs qu'ils apportent et cherchent à s'inscrire dans un processus d'apprentissage mutuel. La co-production des savoirs se fonde sur la réduction maximale des asymétries d'autorité dans les rapports entre les différents savoirs et les différents statuts sociaux en présence. Elle dépasse la seule recherche de la satisfaction des intérêts particuliers par la définition d'un objectif partagé visant un « bien commun ». Le Groupement de recherche du CNRS « Participatory Action Research and Citizen Sciences » (GDR PARCS $^{4}$ ) apparaît dans ce contexte institutionnel et s'intéresse principalement aux pratiques ayant un haut degré d'intensité et de précocité dans la collaboration entre chercheurs et non-chercheurs. Nos objectifs sont d'expérimenter et de développer des méthodologies de Recherche Action Participative (RAP) en adoptant les fondements de la co-production des connaissances, des savoirs, des normes et des innovations.

\section{La Recherche Action Participative comme modalité d'intervention du GDR PARCS}

Ces pratiques de co-production des savoirs s'inscrivent depuis les années 2000 dans un cadre institutionnel qui leur offre une reconnaissance et des opportunités de développement. Elles dépassent le cadre théorique définit par la production de connaissances en mode 2 [GIB 94], [NOW 01] développé au sein de l'espace européen de la recherche, orientation reprise également dans la programmation de l'ANR. L'intérêt de la Recherche Action Participative (RAP) est d'aller au-delà en

\footnotetext{
${ }^{3}$ II distingue le modèle 3 de la co-production des savoirs du modèle 1 de l'instruction publique et du modèle 2 du débat public.

${ }^{4}$ www.gdrparcs.fr 
intégrant dans la réflexion, des parties prenantes issues de la société civile pour effectuer un travail de recherche plus transversal. Différents appels à projets de recherche ont ainsi visé à financer exclusivement des programmes adoptant des modes de collaboration entre chercheurs et acteurs de la société civile non marchande (associations, collectivités territoriales...) $)^{5}$. Une pluralité d'acteurs institutionnels et associatifs cherche à appréhender en France le foisonnement des démarches de collaboration entre chercheurs académiques et acteurs de la société civile non marchande [STO 13], [HOU 16], [ALL 17]. Toutefois, la variabilité sémantique visant à qualifier ces phénomènes illustre la nécessité d'approfondir les travaux de recherche axés sur la caractérisation des pratiques existantes. Le ministère de l'Environnement qui a développé le programme REPERE ${ }^{6}$ pour financer des projets de recherches participatives, a expérimenté la mise en place d'un dispositif d'accompagnement dont l'objectif était de permettre l'engagement d'une démarche réflexive à partir de l'observation des processus de collaboration. Malgré cette démarche, un manque se fait toutefois ressentir lorsqu'il s'agit de saisir les résultats de telles dynamiques. Si les incitations institutionnelles se font de plus en plus explicites $^{7}$ et si les initiatives plus ou moins formelles de collaboration se multiplient, la recherche a pour l'instant très peu questionné les effets de ces démarches et ne parvient que partiellement à qualifier la «co-production des savoirs» à leur aboutissement [FIO 17]. L'un des objectifs prépondérant que s'est donné le GDR PARCS est de travailler cette question de la co-production par le déploiement d'une ingénierie relative à la mise en place de processus de RAP. Le GDR PARCS a été créé en 2013 au sein de l'INÉE ${ }^{8}$ du CNRS avec la volonté de tenir compte à la fois des enjeux de participation, de la résolution d'un problème concret et de la production de connaissances en proposant des outils et des méthodes (via des formations), des conseils aux chercheurs qui souhaiteraient les appliquer (missions d'expertise) et une réflexion de fond sur les démarches participatives. Ses membres œuvrent collectivement à l'amélioration des techniques participatives et à la diffusion de cette approche. Cette ingénierie doit être adaptée aux domaines d'application visés et s'appuie sur des méthodologies visant à garantir la co-construction des problématiques et des réalisations de recherche. Le but final est l'émergence d'innovations sociales susceptibles de répondre aux problèmes de terrain à partir de la co-production des connaissances et des savoirs obtenus. Il parait essentiel aux membres du Groupement de penser les conditions rendant possible l'appropriation par les acteurs concernés de ces co-productions. En d'autres termes, la RAP s'appuie sur la synergie entre les trois piliers que sont la recherche scientifique, l'action et la participation. Sur le plan de la recherche, la méthodologie est orientée vers un travail «avec » les parties prenantes, et non «sur eux» ou "pour eux ». Il se pose alors la question de la place des chercheurs dans un tel processus, donc de leur posture. Sur le plan de l'action, il s'agit de mener des expériences où les parties prenantes ne sont pas prises comme objet d'enquête mais de garder une parité dans la prise de décision et le cadrage du projet dans l'ingénierie à développer. Il est intéressant à ce niveau de combiner l'approche francophone de la psychosociologie et l'approche pragmatique anglo-saxonne. Sur le plan de la participation, elle s'inscrit dans une dynamique de réappropriation par la population de ses modes de vie au travers d'une diversité de formes consensuelles ou de formes plus conflictuelles. La difficulté principale réside dans l'équilibre entre ces piliers [CHE 13].

Les domaines d'intervention principaux de la RAP aujourd'hui sont de cinq ordres: (i) les organisations et le monde du travail avec une grande part d'analyses sociotechniques et de stratégies organisationnelles; (ii) les relations entre individus et société avec les différents courants de la psychosociologie; (iii) l'éducation avec les pédagogies critiques et les études sur le féminisme ; (iv)

\footnotetext{
${ }^{5}$ Par exemple, les programmes portés par des conseils régionaux tels que les Partenariats Institutions-Citoyens pour la Recherche et I'Innovation (PICRI, île-de-France) et Chercheurs-Citoyens (Nord-Pas de Calais).

${ }^{6}$ Réseau d'Échange et de Projets sur le Pilotage de la Recherche et l'Expertise - http://www.programme-repere.fr/

${ }^{7}$ On peut également évoquer ici l'organisation par le CNRS, ATD Quart-Monde et le CNAM le $1^{\text {er }}$ mars 2017 à Paris du colloque «Construire les savoirs avec tou.te.s ? Recherches participatives avec les personnes en situation de pauvreté ».

${ }^{8}$ Institut Écologie et Environnement - http://www.cnrs.fr/inee
} 
l'intérêt porté aux communautés dans un contexte international; (v) l'engagement du public avec la sociologie de l'intervention, la démocratie délibérative et les sciences citoyennes [CHE 13].

La base de travail du GDR PARCS se situe préférentiellement dans le dernier domaine d'intervention tout en élargissant le champ de la RAP à des méthodologies liées aux recherches participatives (co-production des connaissances et des savoirs) et aux sciences participatives (collecte de données et analyses collectives). Les problématiques développées sont d'ordre sociotechniques, en lien avec les domaines du développement soutenable (17 ODD de $\left.1^{\prime} \mathrm{ONU}^{9}\right)$ et des transitions (écologique, énergétique, alimentaire...), domaines intéressant l'INEE. L'originalité du GDR PARCS est de se concevoir comme un laboratoire hors-mur qui réunit des chercheurs de disciplines différentes: sciences expérimentales (physique, biologie, écologie, médecine), sciences sociales (sociologie, géographie, éducation, STS), ingénierie (statisticiens, praticiens, agronomes) et société civile (associations, ONG, bureau d'étude, ESS, entreprises). Comme cette diversité le laisse entrevoir, l'objectif est de dépasser le stade d'extraction des observations ou connaissances du citoyen afin d'alimenter la base de données du chercheur pour aller vers la co-construction des projets de recherche caractérisée par une collaboration permanente et l'élaboration conjointe de solutions.

\section{L'intérêt pour le GDR PARCS de poursuivre ses travaux en RAP}

Le GDR PARCS est actuellement en phase de renouvellement après quatre années de fonctionnement. Nous synthétisons et analysons ici les travaux menés au sein du GDR pris comme cas d'étude ( $70 \%$ chercheurs $-30 \%$ associatifs, $50 \%$ de pratiquants RAP sur projets en cours). Nous avons réalisé une enquête en août 2017 en ligne auprès de ses membres et à partir d'exemples concrets (tourisme responsable, nouvelle pédagogie...) [BLA 17]. Vingt-cinq membres sur quarante ont répondu au questionnaire qui comportait sept questions. Ces dernières étaient orientées vers les formations organisées au sein du GDR et étaient destinées à connaître les apports, les attentes, les résultats, les interrogations et les problèmes lors de la mise en œuvre de projets RAP.

Il faut d'abord rappeler que les membres du GDR ont formulé assez tôt le constat de l'importance du « comment faire participer le public ?», et donc de l'ingénierie du processus RAP. Une des premières actions a été la réalisation de séquences de formations annuelles RAP permettant quelques avancées dans son utilisation au sein des projets répartis sur le territoire. Grâce à l'accompagnement, à l'animation de formations et aux outils et concepts développés par Jacques Chevalier de l'Université de Carleton à Ottawa ${ }^{10}$, quatre formations de deux à trois jours chacune et une école thématique de cinq jours ont été organisées. Ce sont plus de cent quatre-vingt personnes en tout qui ont été formées en France dont quarante étudiants sur vingt jours de formation. Les participants étaient issus principalement du monde académique mais aussi du monde associatif, plus rarement de bureaux d'étude et d'entreprise. La plupart des participants ont suivi au moins deux formations, ce qui dénote un engagement jugé fructueux. Ces formations leur ont permis principalement l'acquisition «d'une diversité d'outils utiles pour la co-construction et l'accompagnement de projets de recherche », une forme de réflexivité et une mise en réseau. Les projets de recherche menés par les participants sont assez divers. Ils viennent de disciplines variées (biologie marine, agro-foresterie, anthropologie, agriculture, sociologie, sciences de l'éducation, médiation scientifique) reflétant la diversité des champs disciplinaires identifiés dans la littérature. Si la mise en œuvre reste «modeste » pour certains, elle permet principalement « un mode d'animation de séances de réflexion collective pour les projets de recherche participative, la co-conception ». La mise en œuvre des piliers de la participation et de l'action semble plus aisée que la production de recherche (au sens académique) ; il s'agit d'ingénieries de projets qui devraient déboucher à terme sur la production de résultats de recherche (publications

\footnotetext{
${ }^{9}$ http://www.un.org/sustainabledevelopment/fr/objectifs-de-developpement-durable/

${ }^{10}$ www.sas2.net
} 
dans des revues etc). Seuls quelques membres appliquent la RAP dans tous ses aspects et régulièrement.

Les formations introductives et avancées ont ainsi permis aux membres du GDR et à la communauté scientifique française élargie de monter en compétence sur les outils de la RAP, d'introduire plus de participatif dans les sites de sciences participatives existants (pour aller au-delà de l'extraction des données) et surtout de contribuer au développement d'une science et d'une ingénierie de la participation.

Plus globalement, le fonctionnement du GDR a permis à ses membres d'étendre leurs réseaux vers d'autres domaines et d'autres disciplines. Il a permis aux chercheurs de se rapprocher des acteurs de la société civile et aux structures associatives de rencontrer le monde académique. Cette première structuration d'un réseau français de la Recherche Action Participative, autour d'une problématique de développement de compétences, apparaît comme l'un des principaux résultats du fonctionnement du GDR.

Parallèlement, l'organisation de formations et le partage d'expériences ont comblé le besoin de connaissances et de compétences dans la pratique et les outils des approches participatives. On peut noter que certains participants ont trouvé de nouvelles opportunités dans le GDR, tandis que d'autres ont pu y développer leurs acquis. La possibilité de prendre du recul et l'ouverture vers des approches performantes constituent ainsi des apports importants que les membres du GDR ont mis en avant. Le GDR prévoit des formations RAP qui soient dorénavant plus dans l'échange de pratiques et l'analyse des processus et des transformations sociales induites par la RAP. Les emboîtements de techniques, les dispositifs stables pouvant être reproduits auprès de publics différents seront privilégiés.

À la suite des formations annuelles, le GDR a créé un réseau d'entraide au design et à la coanimation d'événements et d'ateliers participatifs. Les membres se sont aidés pour co-concevoir un design et ont pu aussi prêter main forte à l'animation. Ce sont soit des projets en cours qui ont été accompagnés, soit des projets nouveaux qui ont vu le jour grâce à ce réseau d'entraide. Tous les projets suivants ont bénéficié de ce réseau : intégrité scientifique, posture, valeurs, transition énergétique, ERC (Eviter, réduire et compenser), qualité de l'air, cohabitation homme/faune sauvage, analyse citoyenne des données, déchets plastiques et tortues marines... L'accompagnement du GDR dont ils ont bénéficié est fonction de l'aisance avec laquelle les porteurs manipulent les outils de la RAP, le degré de maturité et de réflexion sur la thématique qui a nécessité le recours à cette approche. Cet accompagnement est de plusieurs ordre :

- Aide au design d'ateliers dans un cursus en cours de doctorant en bourse Cifre (Ministère/entreprise) qui souhaite consulter ses interlocuteurs locaux (ERC, territoires).

- Co-animation d'ateliers qui visent à concevoir et à tester un dispositif (intégrité scientifique, valeurs).

- Formation à la RAP d'un collectif ou d'une équipe qui vient d'être financée et qui souhaite acquérir un même langage et faciliter le dialogue entre les différents partenaires du projet. C'est le cas de Valuebugs ${ }^{11}$ financé par le programme Co Create de Innoviris porté par la Région Bruxelloise.

-Co-conception et co-pilotage d'un projet initié dans le cadre du GDR et mis dans les priorités dès le démarrage du réseau en 2013. C'est le cas du projet «Analyse Citoyennes des Données » (ACD) qui nous amène à revoir en équipe le concept même de sciences citoyennes et de sa composante de recherche extractive.

Le projet ACD a été entièrement conçu par un collectif issu du GDR composé de chercheurs (CIRAD, INRIA, INRA, CNRS) impliqués dans des projets associatifs participatifs à visée naturaliste

\footnotetext{
${ }^{11}$ http://www.ulb.ac.be/recherche/presentation/fr-cocreate.html
} 
(Tela Botanica, OrchisSauvage, Observatoire des Saisons, Cybelle Planète). Il s'est inspiré des travaux de recherche réalisés autour d'un web collaboratif ou "géocollaboratory " nommé ABORINET ${ }^{12}$ visant à développer des recherches en ligne à distance entre 200 initiatives de tourisme autochtone à travers le monde [BLA 12]. Le collectif s'est donné les moyens d'analyser les différentes options développées par des projets de sciences participatives en Amérique du Nord et de repenser leurs propres sites pour mieux répondre aux attentes des citoyens pourvoyeurs de données. Il s'agit en amont, de la co-construction des questions de recherche avec le citoyen et de la refonte du protocole, et en aval de la possibilité d'analyser et d'interpréter des jeux de données collectées collectivement et d'alimenter les pistes d'action envisagées. Les outils du numérique facilitent la collecte des données à grande échelle mais ne suffisent pas à répondre aux attentes des citoyens. C'est en combinant les outils de la RAP en présentiel (ateliers) et en virtuel (site internet intégrant des nouvelles fonctionnalités) que nous arrivons à fidéliser nos pourvoyeurs de données mais surtout à créer une communauté pensante et solidaire.

C'est au cours de l'école thématique du GDR en avril 2016 qu'est né le projet «intégrité scientifique ». Il a été conçu «sur mesure » avec Jacques Chevalier en réponse à une demande de renforcement de l'intégrité scientifique d'un chargé de mission d'un organisme de recherche français. Il s'agissait de développer une réflexion au sein de la communauté scientifique sur les manquements observés dans la recherche, les valeurs sous-jacentes qui animent les chercheurs et les pistes d'actions à développer. Le dispositif consiste à animer une série d'ateliers en RAP auprès de publics variés (Doctorants d'Agreenium et d'Ecoles Doctorales, chercheurs en cours d'évaluation) et de faire générer par les participants ces éléments plutôt que de les leur imposer sous la forme de formations ou de présentations plénières. Les 4 ateliers animés depuis 2017, reproduits sur le même modèle, ont eu lieu à Montpellier et en Guadeloupe et ont permis de rassembler des données qualitatives et quantitatives qui ont vocations à être source d'inspiration et de référence pour les formations à venir au sein des écoles doctorales, des équipes de recherche et dans le cadre des évaluations quadriennales des laboratoires [FEL 17] $]^{13}$.

Dans les deux cas, l'approche RAP a été déterminante pour apporter une autre dimension à la coconception d'un programme de recherche, à l'exploration de nouvelles pistes visant l'éthique, la solidarité et le vivre ensemble et la création de nouvelles façons de se réapproprier des concepts ou de développer des dispositifs plus efficaces que les interventions ou formations traditionnelles.

\section{Questions et enjeux autour de la Recherche Action Participative}

Une représentation traditionnelle du mode de production des connaissances scientifiques associe fondamentalement la production de savoirs à l'œuvre d'un personnage savant. Cette personne, à partir de ses capacités personnelles et d'une opération de synthèse, se dit et se voit en capacité d'assembler des informations, d'innover intellectuellement, de générer des activités de synthèse, enfin, d'être en mesure de présenter des explications/hypothèses porteuses de sens sur de grandes interrogations sociétales ou philosophiques. Cette représentation est néanmoins complétée aujourd'hui par celles de modes de production plus collective d'équipe de recherche si l'on pense à des travaux de recherche fondamentale en sciences expérimentales. Dans tous les cas, il s'agit de personnes ayant des aptitudes et des capacités spécifiques, ce sont des personnes formées par et pour la recherche et œuvrant fondamentalement au développement des sciences au sein d'institutions universitaires ou affiliées à celles-ci.

Une représentation moins traditionnelle du processus de production des connaissances associe cette capacité à toutes les personnes dotées d'aptitudes intellectuelles: qu'importent leurs origines, qu'importe leur statut social. Selon cette représentation, toutes les personnes d'une société ou d'une

\footnotetext{
${ }^{12}$ http://aborinet.cefe.cnrs.fr

${ }^{13}$ Ces ateliers répondent au programme européen SwafS-02-2018 : Innovative methods for teaching ethics and research integrity 
communauté ont la capacité de participer au processus de production des connaissances. Toujours selon cette représentation, il apparaît évident que toutes les personnes qui le font ne verront pas nécessairement leur apport être reconnu puisqu'elles n'auront pas nécessairement accès aux dispositifs qui rendent possible la diffusion et l'institutionnalisation de leurs idées, de leurs pratiques. Ces personnes n'auront pas non plus toutes les compétences nécessaires pour rendre explicites les nouvelles connaissances qu'elles ont générées. Enfin, leurs idées circulant moins bien ou pas du tout, ces personnes ne pourront pas facilement soumettre leurs hypothèses ou explications au test de la preuve, c'est-à-dire au processus d'évaluation par une communauté scientifique de leurs idées, de leurs théories ou de leurs démonstrations.

L'intérêt de la RAP se situe au croisement de ces deux perspectives. Ce type de recherche reconnaît l'importance de combiner les expertises cognitives, les connaissances expérientielles et les savoirs émanant de chercheurs universitaires et de représentants de différents milieux professionnels ou citoyens. Il ne remet pas en cause la raison d'être de la recherche fondamentale, ni celle de la recherche appliquée telle que promue classiquement par l'institution universitaire. Mais les travaux récents des sciences et des recherches participatives tendent à montrer qu'elles ont également leur place. Ces différentes manières de faire de la recherche doivent être reconnues et soutenues à leur juste valeur. Le mode « avec » rappelle que pour travailler ensemble, il importe de mettre en place des conditions pour faciliter cette rencontre. Il s'agit d'ouvrir le domaine de la science à d'autres professionnels, à d'autres intellectualités qui ne font pas de la science leur premier métier. Le fait de prendre en compte la richesse intellectuelle des non universitaires et de mettre en place des dispositifs de construction conjointe des connaissances constitue un saut qualitatif important.

Dans cette veine de pensée, les travaux de Berger et Luckmann [BER 66] sur la construction sociale de la réalité, ont permis d'ouvrir le débat sur la complexité du processus de production du vivre ensemble. Les travaux de Latour et Woolgar [LAT 88] et ceux de Callon [CAL 92] ont poursuivi sur cette lancée en montrant comment le processus de recherche s'enrichit lorsqu'il met en scène une variété de personnes et d'objets. Cet ensemble d'humains et de non humains constitue un laboratoire producteur de nouveaux savoirs, de nouvelles pratiques. Dès lors, il devient important tant de favoriser la rencontre entre les humains et les non humains que de faciliter leur association dans le travail de production de nouveaux savoirs et de nouvelles pratiques.

\section{Des questions en suspens}

Pour des chercheurs œuvrant dans un cadre classique de recherche fondamentale n'impliquant pas de mises en relations formelles avec des acteurs sociaux, le fait de travailler de concert avec des cochercheurs non universitaires sur des questions très liées aux besoins et aux intérêts de groupes sociaux peut représenter un problème.

- Est souvent posé la question de la pertinence et de la légitimité de travailler dans un cadre qui donne l'impression d'être au service de l'acteur partenaire de la recherche. La science doit-elle se mettre au service des acteurs sociaux et courir le risque de devenir « utilitariste»?

-Du point de vue scientifique, est posée une question miroir : celle de la scientificité d'une telle démarche. Qu'en est-il de la rigueur scientifique lorsque l'agenda politique semble omniprésent? Les chercheurs pratiquant la recherche partenariale accordent-ils préséance à l'engagement social sur la quête de vérités scientifiques?

Il est certes difficile de répondre dans l'absolu à ces questions mais les résultats obtenus ont permis une couleur et des avenues cognitives différentes de celles engendrées par et dans un processus classique de recherche, et ce pour différentes raisons. Notons que contrairement aux méthodes classiques, la Recherche Action Participative, reconnaît le risque de l'utilitarisme ou de la dérive. Le chercheur utilisant des méthodes classiques est lui aussi influencé par les processus de socialisation (genre, origines, classe...), les modalités de financement, ses propres désirs et valeurs (faire sa marque, être reconnu...). Ce chercheur est moins conscient de cette subjectivité et de l'utilitarisme qu'il dessert 
inconsciemment. Il est plus disposé à penser que ce type de recherche est en soi « objectif » et dénué de toute influence morale ou marque politique [PES 03].

Premièrement, force est de constater que l'objet de recherche, lorsqu'il est défini conjointement, met en scène des préoccupations différentes de celles portées par un chercheur. Par exemple, la problématisation et la formulation de la question de recherche définie de façon coopérative mobilise des intérêts pluriels de recherche et exige un approfondissement cognitif centré tant sur la systématisation de savoirs que sur le passage à l'action. L'éventail des questions et enjeux soulevés par les parties peut requérir la contribution de savoirs disciplinaires distincts et ouvre, dans ce cas, sur une forme d'interdisciplinarité. Il y a donc, par la diversité des préoccupations, des points de vue, des savoirs expérientiels, une orientation particulière et donc une valeur différenciée des résultats obtenus par rapport à ceux qui découleraient d'une recherche sur un « thème similaire » qui serait réalisée de façon non partenariale. Il est vrai que cet apport oriente le travail de problématisation, et ce d'une façon qui modifie l'angle de cette dernière. N'en est-il pas ainsi aussi lorsqu'une problématisation de recherche est construite à partir d'une approche multidisciplinaire impliquant une diversité de chercheurs? En fait, une recherche conduite par plusieurs chercheurs non soumis à une autorité hiérarchique et travaillant de façon collégiale pourra déboucher sur des interprétations différentes en fonction des identités propres aux chercheurs membres de l'équipe.[VAS 02].

Deuxièmement, tout au long de la réalisation de l'activité de recherche, les mises en dialogue entre les parties prenantes assurent une validation en temps réel du traitement des données (collecte et analyse). Le groupe de discussion ainsi constitué fait office de laboratoire ou prennent place des actions de médiation, de traduction $[\mathrm{CAL} 91]^{14}$, d'interprétation et d'appropriation sur le contenu même des données. Ce type de travail implique différentes entrées et surtout différents points de vue sur la réalité et son fonctionnement. Au sein de l'espace collaboratif constitué, le groupe en discussion agit de façon transactionnelle au sens de John Commons [COM 34]. Il repose sur une négociation et ventilation des rôles et des responsabilités, des pouvoirs et des capacitations. Il est parfois source de tensions. Il permet des apprentissages mutuels et un enrichissement de la qualité des analyses. Ce point représente une distinction nette et très caractéristique du processus de co-construction disciplinaire ou interdisciplinaire prenant place entre chercheurs au sein d'une démarche classique de recherche. La distinction prend moins l'aspect d'un travail réunissant des personnes d'horizons différents, comme c'est souvent le cas dans une situation de recherche classique où du travail en commun prend place entre des chercheurs d'horizons disciplinaires différents [MON 05], que dans la mise en relation avec des acteurs (professionnels ou citoyens) travaillant à partir de logiques épistémiques, politiques et morales différentes.

Enfin, il apparaît clairement que la conduite d'activités de Recherche Action Participative pose des questions éthiques et politiques sur :

- la façon de produire des connaissances avec ou sans la présence d'une posture permettant la démocratisation du processus de production et de valorisation des savoirs en associant l'ensemble des parties prenantes concernées à l'activité de co-construction de nouveaux savoirs ;

- la nature de l'impact de cette méthode sur la configuration ou la reconfiguration des disciplines scientifiques concernées par les savoirs ainsi co-produits ;

- l'impact de cette posture et de ses révélations, en termes de nouveaux savoirs, sur le développement futur des populations, des organisations, des secteurs ou des territoires concernés ;

\footnotetext{
${ }^{14}$ Traduction au sens défini par la théorie de l'acteur-réseau où l'action de traduire signifie mettre en relation et en interaction des acteurs provenant d'horizons différents pour les aider à travailler ensemble un problème ou une question. II ne s'agit pas seulement d'aider à passer de l'information, mais à créer dans et par l'interface communicationnel de la nouveauté, de l'innovation.
} 
- finalement, la reconnaissance ou non de l'importance de la «science ouverte » dans le développement du savoir et la transformation des sociétés. En misant sur une pleine reconnaissance de cette importance, il serait alors approprié d'adapter l'écosystème scientifique à cette posture et de doter ce dernier de politiques publiques et sectorielles conséquentes.

\section{Les enjeux soulevés par la Recherche Action Participative}

Deux enjeux se dégagent de nos réflexions. Le premier tient à une pleine et entière participation des parties prenantes dans les dispositifs de RAP.

Cette nouvelle scène demande une clarification et une mise en transparence des rôles joués et des fonctions occupées en termes de types :

- De leadership : pour assurer que les règles de l'art soient respectées et que la co-production ne soit détournée d'une certaine objectivité, que la critique ait toujours sa place.

- De traduction : pour assurer la présence des différents points de vue concernés dans et par une mise en dialogue des savoirs portés par les différentes parties prenantes autour d'une question ou d'un enjeu.

- D'intéressement : pour permettre l'intégration des parties concernées.

- De médiation : pour faciliter le développement de compromis et le travail de négociation.

- De non-négociabilité : pour savoir quand mettre fin à des opérations de construction conjointe lorsque le processus exige un repli des parties prenantes sur elles-mêmes.

- D'écoute : pour faciliter l'insertion de ce qui à première vue peut déconcerter ou remettre en question.

- De modestie : pour accepter que des non chercheurs mettent à jour des clés permettant de déverrouiller le processus d'émergence de nouvelles compréhensions, de nouvelles connaissances.

-De suivi : pour assurer, par le transfert et la mise en liaison, que les nouveaux ou d'anciens savoirs puissent cheminer auprès des publics concernés.

Si les différentes personnes impliquées ne sont pas en mesure de bien exercer leurs rôles, le processus peut facilement dévier des objectifs assignés aux pratiques de Recherche Action Participative.

- À un extrême du processus : les partenaires peuvent uniquement servir d'alibis ou de terrain pour faciliter la réalisation de processus de recherche ou de formation complètement contrôlés par l'agenda universitaire.

- À un autre extrême : les universitaires peuvent se voir censurés par des acteurs qui contrôlent les objets abordés et les données traitées d'une façon telle que la production de connaissances en arrive à cerner des intérêts très limités, idéologiquement orientés et peu collectivisés.

Le deuxième enjeu tient à la responsabilité claire qu'ont les acteurs de la société civile de devoir s'approprier les rudiments de la culture universitaire de recherche lorsque ces personnes acceptent de travailler en partenariat, bien que ce travail d'acculturation doive se faire dans les deux sens. Il est plus difficilement observable au sein des acteurs non scientifiques qui sont habitués à travailler à partir de transactions contractualisées. Dès lors, le danger d'instrumentalisation de la science par l'implication d'acteurs non chercheurs est celui de ne voir ceux-ci s'intégrer que partiellement à une démarche partenariale, générant un déficit de participation. Tous les éléments cognitifs découlant d'une recherche ne seront pas alors nécessairement traités ou abordés si les partenaires ne font pas preuve d'ouverture à cet effet. De plus, il arrive souvent, une fois le projet terminé, lorsque la collaboration avec l'équipe universitaire de recherche leur semble moins nécessaire pour le développement de leur organisation, que ces acteurs considèrent avec moins d'intérêt d'autres activités de recherche. De manière parallèle, 
l'intérêt pour la collaboration peut également être remis en question par les universitaires lorsqu'ils s'approprient l'objet d'étude sans renouveler la démarche participative.

En corollaire, les chercheurs universitaires ont aussi un devoir d'accompagnement à réaliser, et ce dans les limites de leurs fonctions professionnelles. D'où l'intérêt de développer des dispositifs spécifiques avec un soutien de personnels formés à la traduction/médiation active. L'accompagnement peut prendre au moins trois formes complémentaires.

- Une première, consistant à générer un espace collaboratif de travail pour toutes les étapes de la collaboration : de la co-construction du devis de recherche à la co-production du ou des rapports, en passant par une co-analyse des données ;

- Une deuxième, en appuyant la réalisation d'activités de diffusion ou de transfert, l'idée étant de maximiser les retombées en termes d'appropriation par le ou les milieux concernés.

- Une troisième, fondée sur une évaluation de toute la démarche de co-construction de la recherche qui appuierait une analyse réflexive sur l'ensemble du processus et sur le sens et la portée des connaissances produites.

\section{Trois grands défis à prendre en considération}

Pour les chercheurs, ainsi que pour l'institution universitaire concernée, un premier défi est d'assurer une pleine et entière reconnaissance des activités participatives de recherche, de formation ou d'accompagnement. Des chercheurs voient souvent leurs travaux dévalorisés parce qu'ils ne répondent pas aux critères normatifs d'évaluation de la production scientifique, lesquels critères ne sont toujours pas ajustés en fonction de cette autre façon de co-produire des connaissances. Il importe de noter que des revues (même de belle réputation) à comité de lecture intègrent des travaux en co-production (avec des naturalistes non-professionnels comme co-auteurs, par exemple) et que l'acculturation réciproque permet à la Recherche Action Participative de suivre ce chemin. Somme toute, des revues spécifiques peuvent être également à créer.

Sortir de cette posture implique de revoir les bases mêmes du financement de la recherche. Ceci :

- permettrait la mise en place de nouveaux types de réseaux de recherche et de partenariats ;

- impliquerait d'allouer des enveloppes budgétaires pour que :

- les partenaires non-académiques puissent bénéficier de ressources financières en appui à leur participation au processus de recherche ;

- les chercheurs accordent plus d'attention à la dimension appropriation et transfert des connaissances.

Sur ce point, l'imaginaire actuel est cantonné dans une logique de l'innovation linéaire où le besoin en recherche donne lieu à une activité de recherche de laquelle découlent des résultats qui sont publiés. La publication est considérée comme un bien à consommer. Dernièrement, un semblant de percée a été réalisé avec l'idée de «mobilisation des connaissances». En fait, par «mobilisation », certains s'en tiennent à des activités de courtage pour rendre accessible des connaissances universitaires auprès de différentes « clientèles ». Si cette fonction est importante, elle constitue tout au plus une des modalités d'action à entreprendre.

Un nouvel imaginaire, misant sur la circularité du processus et des échanges soutenus entre les parties prenantes à chacune des étapes de la recherche, permet un transfert réel et plus dynamique des connaissances. Ce nouvel imaginaire devient une condition porteuse pour assurer aux collectivités une plus grande capacité dans la prise en charge de leur développement. Il reste à identifier les indicateurs pertinents qui permettraient de rendre compte de cette nouvelle réalité. 
Un deuxième défi consiste à approfondir la dynamique du processus participatif de coopération de la recherche et sa diffusion lorsque cette dernière regroupe des acteurs variés.

D'une part, il s'agit de mobiliser de façon plus active et participative les acteurs du terrain dans la problématisation des questions de recherche et dans le montage méthodologique de collecte et d'analyse des données. Il s'agit donc de partager la méthode scientifique avec ces derniers, une culture reposant fondamentalement sur l'articulation entre des éléments d'observation empirique, de réflexion analytique critique et des modèles théoriques de conception de l'ordre des choses.

D'autre part, pour les universitaires, il s'agit de rendre plus explicite la culture de «l'action dans l'urgence» qui constitue la réalité au jour le jour des acteurs sociaux. Transmettre cette culture de l'intervention stratégique soulève la question du rapport que les chercheurs doivent entretenir avec l'engagement politique : jusqu'où doit-on aller, quelles frontières faut-il franchir ou ne pas franchir, et surtout quels garde-fous doit-on mettre en place pour garder le cap de la scientificité ?

Un troisième défi est d'ordre cognitif : la recherche scientifique peut difficilement faire l'économie de sa propre évaluation.

En quoi le fait d'investir dans la Recherche Action Participative permet-il de produire une connaissance plus appropriée du monde dans lequel nous vivons? En d'autres mots, la science doit clarifier la mission sociale qu'elle se donne. Ce processus de clarification représente en soi le «projet développemental de la science», dont les contours sont actuellement flous et les moyens et les ressources pour le réaliser pratiquement absents.

Ce projet scientifique, nous le résumons comme une tentative critique et rigoureuse, toujours renouvelée, de rendre le réel compréhensible, transparent et accessible aux différents publics et de permettre des transformations plus compatibles avec le respect du vivre ensemble et de la soutenabilité.

\section{Conclusion}

Le cadre général dans lequel nous nous situons est celui du développement de la démocratie délibérative qui influence nécessairement le champ de la recherche scientifique et de l'innovation en particulier dans des domaines sociotechniques. Nous nous focalisons sur le nécessaire développement de recherche aux interfaces entre sciences expérimentales, sociales et humaines en intégrant la dimension citoyenne. Nous avons décrit les problématiques spécifiques que soulève la participation citoyenne, les réalisations en cours dans une dynamique internationale forte et nous avons pointé l'émergence d'une forme plus originale basée sur une co-production des connaissances et des savoirs. C'est dans cette émergence que s'inscrivent les travaux du GDR PARCS.

Le GDR PARCS se conçoit comme un laboratoire hors-mur défini comme un lieu d'expérimentation et d'analyse s'appuyant sur des méthodologies de Recherche Action Participative. Son domaine d'intervention est celui de l'engagement de parties prenantes, chercheurs et publics concernés, sur des problématiques de recherches scientifiques vers les domaines transversaux liés au développement soutenable, aux transitions écologique, énergétique, alimentaire et plus généralement aux défis sociétaux définis par la plupart des agendas des politiques scientifiques. La RAP permet d'une part, de concrétiser une société de la connaissance par le développement d'une capacitation citoyenne basée sur la co-production des connaissances, des savoirs et des innovations. D'autre part, elle offre un cadre épistémologique intéressant pour le traitement de problématiques complexes et transversales: association dialogique des sphères de la recherche et de l'action, logique de régulation continue des rapports entre les objets d'étude et les chercheurs qui les étudient, approche intégrée des questions de recherche.

Nous montrons que la médiation, la traduction et l'accompagnement méthodologiques par une ingénierie participative appropriée à une problématique contextualisée est importante et qu'elle tire 
bénéfice d'un cadre stable et pérenne. La difficulté principale réside dans l'équilibre entre les trois piliers que sont la recherche, l'action et la participation. Le développement de structures ou dispositifs convenablement outillés semble nécessaire pour permettre une meilleure articulation entre les équipes de recherche, les praticiens et les associatifs. Ce développement ne pourra pas se faire sans intégrer une dimension éthique, qui se formalise aujourd'hui autour de la question des chartes de la participation.

\section{Remerciements}

Nous remercions le labex CEMEB et le labex DRIIHM pour le financement des formations à la Recherche Action Participative et l'INEE du CNRS pour la labellisation du GDR PARCS et son soutien de 2013 à 2017.

\section{Références}

[ALL 17] ALLISS, Prendre au sérieux la société de la connaissance, Livre blanc, 80p., 2017

[ANA 07] ANADÓN M., La recherche participative. Multiples regards, Presses de l’Université du Québec, Québec, 2007.

[AUD 11] AUDOUX C., GILLET A., « Recherche partenariale et co-construction de savoirs entre chercheurs et acteurs: l'épreuve de la traduction », Revue Interventions Economiques, n43, p. 1-16, 2011

[AUD 15] AUDOUX C., Un possible, impossible, la co-production des connaissances entre science et société. Étude de recherches collaboratives entre chercheurs et acteurs dans le cadre du dispositif « Partenariat Institutions Citoyens pour la Recherche et l'Innovation » (PICRI) en Ile-de-France, Thèse de doctorat en sociologie, Conservatoire national des arts et métiers, 340 p., 2015

[BER 66] BERGER P., LUCKMANN T., The Social Construction of Reality, Doubleday, New-York, 1966

[BLA 17a] BLANGY S., Co-construire le tourisme autochtone par la recherche action participative et les technologies de l'information. Une nouvelle approche de la gestion des ressources et des territoires, L'Harmattan, Paris, 2017

[BLA 12] BLANGY S, DONOHOE H., MITCHELL, S., Developing a geocollaboratory for Indigenous tourism research, Current issues in tourism, Vol.15, nº7, p. 693-706, 2012

[BLO 02] BLONDIAUX L., SINTOMER Y., « L'impératif délibératif. », Politix, Vol.57, n¹, p. 17-35, 2002

[BON 13] BONNEUIL C., JOLY P.B., Sciences, techniques et société, La Découverte, Paris, 2013

[BON 14] BONNEY R., SHIRK J.L., PHILliPS T.B., WIGGINS A., BALLARD H.L., MILLER-RUSHING A.J., PARRISH J.K., « Next step for Citizen Science », Science, Vol.343, p. 1436-1437, 2014.

[BOU 05] BOURG D., BOY D., Conférences de citoyens, mode d'emploi, les enjeux de la démocratie participative, Ed. Charles Léopold Mayer et Descartes et Cie, Coll. TechnoCité, Paris, 2005

[BOU 11] BOURG D., WHITESIDE K., «Écologie, démocratie et représentation. », Le Débat, Vol.164, n², p. 145-153, 2011

[CAL 92] CALLON M., "Sociologie des sciences et Économie du changement technique: l'irrésistible montée des réseaux technico-économiques », dans Ces réseaux que la raison ignore, L'Harmattan, Paris, p.53-78, 1992.

[CAL 98] CALLON M., « Des différentes formes de démocratie technique », Annales des mines, n9, p. 63-73, 1998.

[CAL 01] CALLON M., LASCOUMES P., BARTHE Y., Agir dans un monde incertain. Essai sur la démocratie technique, Seuil, Paris, 2001.

[CAL 91] CALLON M., LATOUR B., La science telle qu'elle se fait. Anthologie de la sociologie des sciences de langue anglaise, La Découverte, Paris, 1991.

[CHA 89] CHAMBERS R., PACEY A ., THRUPP L.A., Farmer first: farmer innovation and agricultural research, Intermediate Technology Publications, London, 1989

[CHE 13] CHEVALIER J.M., BUCKLES D.J. Participatory Action Research - Theory and methods for engaged inquiry, Routledge, London and New-York, 2013

[COH 89] COHEN J., « Deliberation and democratic legitimacy », dans D. MATRAVERS et J.E. PIKE (eds.), Debates in Contemporary Political Philosophy: An Anthology. Routledge, 1989 
[COM 15] COMETS - Comité d'éthique du CNRS, Avis du Comets. Les «sciences citoyennes », 12p. URL: http://www.cnrs.fr/comets/spip.php?article125, 2015

[COM 34] COMMONS J.R., Institutional Economics, The Macmillan Co., New-York, 1934

[COU 15] COUTELLEC L., "Pour une philosophie politique des sciences impliquées - Valeurs, finalités, pratiques », Ecologie \& Politque, n51, p. 17-25, 2015

[DEL 14] DELL'ERA C., LANDONI P., «Living Lab: A Methodology between User-Centred Design and Participatory Design. », Creativity and Innovation Management, 23, p. 137-154, 2014

[DEW 27] DEWEY J., The public and its problems, 1927

[EPS 01] EPSTEIN S., La Grande révolte des malades. Histoire du Sida 2, Les Empêcheurs de penser en rond/Le Seuil, Paris, 2001.

[FEL 17] FELDMANN P., BLANGY S., CHEVALIER J., Participatory Action Research in support of research integrity: engaging the parties concerned, 5th World conference on research integrity (WCRI), p. 122, Amsterdam, 2017

[FIO 17] FIORINI C., «Étudier les pratiques de co-production des savoirs pour interroger leurs effets », Communication lors de l'École thématique de l'IFRIS : Sciences citoyennes? Technologies, politiques et pratiques de la participation, Marseille, 12-15 juin 2017.

[FOU 01] FOURNIAU, Le débat public: une expérience française de démocratie participative, La Découverte, Paris 2001

[GIB 94] GIBBONS M., LIMOGES C., NOWOTNY H., SCHWARTZMAN S., SCOTT P., TROW M., New production of knowledge : dynamics of science and research in contemporary societies, Sage, Londres, 1994.

[GIR 13] GIRARD H, "Démocratie délibérative», dans I. CASILLO, R. BARBIER, L. BLONDIAUX, F. CHATEAURAYNAUD, J.M. FOURNIAU, R. LEFEBVRE, C. NEVEU ET D. SALLES (dir.), Dictionnaire critique et interdisciplinaire de la participation, GIS Démocratie et Participation, Paris, 2013.

[HAB 87] HABERMAS J., Théorie de l'agir communicationnel, t1 et 2, Fayard, Paris, 1987

[HOU 16] HOULLIER, F., Les Sciences participatives en France. Etat des lieux, bonnes pratiques et recommandations, Rapport pour le Ministère de L'Éducation Nationale et de la Recherche, URL: http://www.sciencesparticipatives.com/Rapport, 2016.

[KUL 16] KULLENBERG C., KASPEROWSKI D., «What is citizen science? - A scientometric meta-analysis », PloS ONE, Vol.11, $\mathrm{n}^{\circ} 1, \mathrm{p}: 1-16$

[LAT 88] LATOUR B., WOOLGAR S., La vie de laboratoire - La production des faits scientifiques, La Découverte, Paris, 1988

[LEQ 14] LEQUIN Y-C., LAMARD P., Éléments de démocratie technique, UTBM. Editions, 2014.

[MAN 85] MANIN B., «Volonté générale ou délibération ? Esquisse d'une théorie de la délibération politique », Le Débat, vol. 33, no. 1, p. 72-94, 1985

[MAN 95] MANIN B., Principes du gouvernement représentatif, Calman-Levy, Paris, 1995

[MON 05] MONDADA L., Chercheurs en interaction. Comment émergent les savoirs, Presses polytechniques et universitaires de Lausanne, Lausanne, 2005.

[NOW 01] NOWOTNY H., SCOTT P., GIBBONS M., Re-thinking science: knowledge and the public in an age of uncertainty, Polity Press, Cambridge, 2001.

[PES 03] PESTRE D., Science, argent et politique, INRA Editions, Paris, 2003.

[PES 06] PESTRE D., Introduction aux Science Studies, La Découverte, Paris, 2006.

[PIM 11] PIMBERT M., Participatoryresearch and on-farm management of agricultural biodiversity in Europe, Londres, IIED, 69 p., 2011.

[REV 14] REVEL M., Main Findings Report, Deliverable D2.3, Projet CONSIDER, 7ème Programme-cadre de la Commission européenne, 70 p., 2014

[ROS 06] ROSANVALLON P., La contre-démocratie: La démocratie à l'âge de la défiance, Seuil, Paris, 2006

[RUA 17] RUANO-BORBALAN J.C., La "grande standardisation» et les transformations du savoir dans les sociétés technoscientifiques, Innovations, vol.52, $\mathrm{n}^{\circ} 1, \mathrm{pp} .17-41,2017$. 
[SAV 17] SAVOIA A., LEFEBVRE B., MILLOT G., BOCQUET B., " The Science Shop Concept and its Implementation in a French University », Journal of Innovation Economics \& Management, Vol.2017/1, n²2, p. 97$117,2017$.

[SHI 12] SHIRK J.L., BALLARD H.L., WILDERMAN C.C., PHILLIPS T., WIGGINS A., JORDAN R., MCCALLIE E., MINARCHEK M., LEWENSTEIN B.V., KRASNY M.E., BONNEY R., "Public participation in scientific research: a framework for deliberate design », Ecology and Society, Vol.17, n², p. 1-20, 2012

[STO 13] STORUP B., MILLOT G., NEUBAUER C., La recherche participative comme mode de production de savoirs - Un état des lieux des pratiques en France, rapport de l'association Sciences Citoyennes pour la Fondation de France, $94 \mathrm{p}, 2013$

[VAN 17] VAN DAMME S., Sciences en société - De la Renaissance à nos jours, Documentation photographique - Les dossiers, n8115, La Documentation Française, janvier/février 2017.

[VAS 02] VASILACHIS DE GIALDINO I., «L'interprétation dans la recherche qualitative : problèmes et exigences », Recherches Qualitatives, Vol.31, n³, p. 155-187, 2012. 